\title{
$\operatorname{Six}$
}

\section{QUANTUM OBSERVERSHIP COSMOLOGY}

Conventional non-Everett quantum theory assumes that definiteness in and of the world results from wave-function collapse, and many Quantum Cosmologists try to apply such notions to the universe as a whole. Some Quantum Cosmologists affirm a very intimate relationship between physics and physicists, between observers and things observed. This intimacy is much clearer at the level of microscopic quantum events than at the cosmological level of accounting for the origin of the universe.

Quantum Observership says that if and when a physicist measures for the position of an electron, it then takes a position; if and when a physicist measures for its momentum, it then assumes momentum; since no observer can measure for both simultaneously, it cannot have both together. Sometimes sub-atomic entities behave like particles, sometimes like waves, depending on how observers perceive them. In themselves, apart from being observed, physical entities are nothing; the very concept is meaningless. They have no actuality in themselves; they are only bundles of possibilities.

In Europe, Niels Bohr's "Copenhagen interpretation" of quantum mechanics closely linked observer and observed. In the United States, John A. Wheeler and Eugene Wigner endorsed Strong Anthropic theories of Quantum Observership that emphasize the role of observers in resolving quantum uncertainty and indefiniteness. ${ }^{1}$ Many experiments in quantum physics suggest that unobserved sub-atomic particles in themselves do not exist in a single definite state and that the process of being observed somehow affects what they are. ${ }^{2}$ The two slit experiment indicates that individual photons seem to pass through two (but not three) separate slits at once. Other experiments indicate that the act of observation significantly affects the physical state being observed.

As the story goes, when two spinning particles are generated together, if one is observed to be spinning in one direction, the other instantly acquires the opposite spin, no matter how far away from the first it might be, or how long it has traveled to get there, or even if the decision about how to measure it is made after its departure. If widely separated particles that originated together are observed simultaneously (a difficult if not impossible feat), they do not have time to communicate; but they still have opposite spins. Quantum mechanics rejects as empirically meaningless the realistic position favored by Einstein that particles that originate together have opposite spins from the very outset and continue to have them while unobserved; whenever they are observed the viewer merely sees what has been there objectively all along. Instead, the correct spin supposedly comes into being because it is being observed; observation itself fixes the direction of spin of the second particle. ${ }^{3}$ In Quantum Observership, observation fixes all definiteness. 
In John A. Wheeler's theory of Quantum Observership, physical particles in themselves are nothing more than indefinite probabilistic wave function superpositions of all possible states all at once. According to Quantum Observership, quantum systems take on definiteness or "collapse" only when they are observed. Only measurement by an observer using a macroscopic measuring instrument causes collapse. Wheeler illustrates the essential role of measuring observers in quantum mechanics with this anecdote.

I like the story of the three baseball umpires relaxing over beer one afternoon and comparing notes. One umpire says, "I calls 'em as I sees 'em." The next umpire says: "I calls 'em as they really are." The third one says, "They ain't nothin until I calls 'em."

According to Wheeler, in quantum mechanics "The observer is elevated from 'observer' to 'participator."' $\mathrm{He}$ calls the necessity for observers in the universe the "Participatory Anthropic Principle." According to it, observers are essential for the very existence of the universe and all things within it.

\section{Observers Create the Universe}

Metaphysical Idealism asserts that only minds and their experiences and activities exist; it denies the objective existence of matter, claiming that material things exist only in being perceived. They do not exist in themselves but only in and as the experiences of observers.

Most physics is realistic and assumes that observers have nothing to do with the objective existence of the physical world. By contrast, Quantum Observership is idealistic and presumes that observers have everything to do with the existence of the physical world. According to this theory, unobserved atomic and sub-atomic entities in themselves bear no resemblance to tiny billiard balls. In themselves, they simply cannot be pictured. They are individuated and determinate only when observed. An unobserved world is nothing more than a set of sum-over potentials for all possible worlds and simply does not exist at all as a definite actuality. Eugene Wigner, who stresses the role of consciousness, claims that a definite world is brought into being only when its potentials are observed and measured by conscious beings. ${ }^{6}$ Quantum mechanics thus appears to vindicate John Stuart Mill's dictum that the physical world in itself is nothing more than a set of "permanent possibilities for perception."

In quantum theory as Wheeler and Wigner interpret it, the being of physical entities consists in their being perceived. Wheeler explicitly links quantum mechanics with Bishop Berkeley's "To be is to be perceived" and says that in quantum mechanics "The universe would be nothing without observership as surely as a motor would be dead without electricity." All empirical evidence for the Big Bang is created by observership, and so is the Big Bang itself.' 
Observers create the world by experiencing it and reflecting upon it. Wheeler says that "The observer is participator in genesis,"10 and "Observership brings the universe into being." Thus, the world must be compatible with the existence of observers, for without observers there is no world. By extrapolating backwards from what we perceive to be happening now, we (and not God) create the past, the entire natural history of the universe from the very beginning. So, what caused the Big Bang? We did, says Quantum Observership!

\section{Critique of Quantum Observership}

Quantum Observership, as just described, has serious problems that make it extremely implausible as an ultimate explanatory hypothesis.

\section{A. Incompatibility With Cosmic and Biological Evolution}

The main problem is that Quantum Observership requires our conscious existence long before we actually exist. Conscious observers like us create the Big Bang, but the Big Bang creates all conscious observers! Fred Hallberg rightly indicates that the theory is logically incompatible with plausible scientific accounts of cosmic and biological evolution, including human origins. Hallberg explains:

Wheeler emphasizes that life and consciousness entails both biological evolution, and prebiological physical and chemical evolution ["Genesis and Observership,"pp. 3, 5]. Yet all these forms of evolution involve very specific interactions among highly individuated molecules. So his story seems to require specific, individuated events and entities before consciousness is present to individuate them. ${ }^{12}$

A more plausible and realistic account says that innumerable physical structures and processes, including our galaxy, solar system, and planet existed for billions of years before we or any other conscious observers came into being. Just because observation affects the definiteness of quantum-level events, it does not follow that they have no definiteness at all when not being observed. Throughout fifteen billion years of cosmic evolution, the unobserved physical world in itself was sufficiently definite to do all the things that Big Bang Cosmology says that it did. The unobserved world evolved either from an initial singularity of nothingness or from minimal Planck dimensions through exponential inflation (perhaps) to an astronomically vast cosmos, unpopulated for eons by any conscious observers. Definite physical forces like gravity, the strong nuclear force, the electromagnetic force, and the weak force, emerged from the original Grand Unification. As the universe expanded and cooled, a primordial soup of radiant energy and sub-atomic particles gave way to discrete 
atoms, at first mainly of hydrogen and helium. Under the influence of gravity clouds of these gaseous atoms consolidated to form definite galaxies, stars, and planets. Unobserved atoms of heavier elements, manufactured by nucleosynthesis in supernovas, were sufficiently determinate to produce planets. On at least one planet, earth, definite molecules of carbon dioxide, water, ammonia, methane, and so, on eventually combined to produce DNA in living cells. Unobserved living cells increased in complexity and definiteness and united with other cells to form complex multicellular organisms. After billions of years of evolution, some complex organisms became conscious observers. A few even became quantum physicists and cosmologists!

Yet, according to Quantum Observership, only with the advent of conscious observers and measurers does nature in itself acquire any definiteness at all, even with respect to the instant of its origin in the Big Bang. Without a definite world, no conscious observers exist; but without conscious observers, no definite world exists! The idealistic interpretation of quantum physics founders on this contradiction. John Wheeler recognized the problem, but he did not solve it. ${ }^{13} \mathrm{He}$ only perpetuates the contradiction when he writes, "Beginning with the big bang, the universe expands and cools. After eons of dynamic development it gives rise to observership. Acts of observer-participancy in turn give tangible "reality" to the universe not only now but back to the beginning."14

Richard Rorty says that realism, with its concerns for objective truth and objective existence, makes no practical difference, ${ }^{15}$ but it does. Unless a highly definite universe existed objectively on its own back to the beginning, we would not be here; our being here cannot give the universe and our biochemical environment a reality that our own existence presupposes. Quantum physics is still in its infancy, but one way or another it must overcome the paradoxical antirealism of Quantum Observership.

Quantum physicists disagree on whether the physical world is completely indeterminate in itself, or only partly so. The complete indefiniteness of the Quantum Observership interpretation is extreme. In a less radical Critical Realism, the physical indefiniteness of nature is only partial; the sensory modalities of conscious observers add some kinds and degrees of definiteness to things perceived, but not all of it.

Critical Realism can resolve the paradoxes and puzzles of Quantum Observership and still preserve the genuine advances of quantum physics, severed from the pretentious Idealistic Metaphysics with which it is too often associated. If physical things in themselves are individuated and definite to a high degree, but not completely so, conscious observers would not be required to structure the physical world. The sensory modalities and mechanisms of conscious observers may add something, but not everything, to what is given perceptually; observers do not create what exists in itself. A partly realistic position must try to tell us which is which-which properties are objective, and which are mind-dependent. 
Realism says that a largely determinate physical world exists in itself apart from conscious observation; we perceive certain sensory properties because physical things actually have them and cause us to perceive them. True perceptions accurately reflect objective properties, but illusions and false ones do not. True beliefs accurately describe objective realities, but false beliefs characterize them incorrectly. Realism comes in many varieties. For Naive Realism, objectively existing things have all of the properties that we perceive them to have; but a long tradition of Critical Realism dating back to the Greek Atomists, and resurrected by the originators of modern natural science in the seventeenth and eighteenth centuries, takes a less extreme view. Critical Realism has many contemporary defenders. ${ }^{16}$ It traditionally asserted that "secondary" properties of perceived objects like their color, taste, odor, sound, and perceived temperature are "subjective" or mind dependent; they are added to our percepts by our own creative perceptual modalities and do not exist objectively in nature itself. However, the "primary" physical, spatial, or spatiotemporal properties of things like their size, shape, weight, position, resistance, and motion exist both in our perceptions and objectively in things perceived. Primary properties are the metric or mathematically measurable and quantifiable characteristics of physical things, both in themselves, and as perceived. Our attempts to observe or "measure" quantum-level occurrences always changes their properties, but this does not imply that they have no properties at all when we are not observing them.

In idealistic Quantum Observership, primary qualities are just as minddependent as secondary qualities. Idealistic interpretations of quantum mechanics may even border on Positivism, arguing that it is meaningless to postulate the objective existence of any unobserved physical entities and processes in themselves, even their primary properties, because, obviously, we can never observe or even imagine observing such things. As Werner Heisenberg indicated in his Philosophical Problems of Quantum Physics,

In modern physics, atoms possess geometrical qualities in no higher degree than color, taste, etc. The atom of modern physics can only be symbolized by a partial differential equation in an abstract multidimensional space. Only the experiment of an observer forces the atom to indicate a position, a color and a quantity of heat. All the qualities of the atom of modern physics are derived, it has no immediate and direct physical properties at all, i.e. every type of visual conception we might wish to design is eo ipso faulty. Quantum theory made the atom into something inaccessible to our senses or our imagination, unlike objects within our daily experience. An atom or, more correctly, an electron no longer displays 'in itself' ('an sich') even the simplest geometrical and mechanical properties but it shows them only to the extent to which they can be made accessible to observation by external interference. ${ }^{17}$ 
In 1954, Wolfgang Pauli attacked the "hidden variables" of Einstein's physical realism by saying that "One should no more rack one's brain about the problem of whether something one cannot know anything about exists all the same than about the ancient question of how many angels are able to sit on the point of a needle."'18 Quantum Positivism regards all speculation about what things are when we cannot observe or know them as meaningless.

Actually, we should rack our brains about whether the physical stuff of the world existed objectively in relatively determinate physical states for billions of years before conscious observers evolved. This must be so, or we physical, biological, and embodied observers would not exist! Quantum Observership's metaphysical dogma that atomic and sub-atomic physical entities are nothing more than infinite sets of indeterminate possibilities until they are consciously observed must be renounced, along with Idealistic Metaphysics, if we are to have a plausible scientific account of cosmic and biological evolution.

The obvious solution is that quantum entities in themselves are not just infinite sets of potentialities for definiteness. Quantum Critical Realism holds that they contain within themselves a high degree of definiteness inherited from the past, combined with a finite set of potentials or possibilities for further selfdetermination. Real wavicles are both relatively determinate particles as well as waves, not just totally indeterminate "wave functions" alone.

\section{B. Idealism vs. Realism}

Idealistic interpretations of quantum physics must answer this fundamental question: What causes observers to have sensory experiences? For the realistic dualist or materialist, matter does the job. For Bishop Berkeley, God, not matter, produces all our sensations. For Kant, totally unknowable things or realities in themselves (which are not supposed to cause anything because only appearances can be causes) cause our sensory experiences. For the skeptic David Hume, our sensations are of "unknown origin."

Quantum Observership cannot explain how infinitely complex sets of pure possibilities, totally lacking the power of actual beings, can act upon the sensory organs, nerve cells, and brains of living biological observers like us and cause us to perceive one definite and common world. In fact, it cannot account for the objective existence of sense organs, much less cells, and brains! How can the absolutely indeterminate bring about the partly or completely determinate? How can pure possibilities be efficient causes? How can living biological observers have definite sensory organs, nerve cells, and brains if the unobserved physical world has no definiteness in itself? Quantum Observership has no good answers. In Idealistic Metaphysics, nerve cells and brains exist only as relatively infrequent objects of perception, but not in themselves. In light of what contemporary physics has revealed, how should we conceive of the physical or material world? 


\section{i. Critical Realism and Quantum/Relativity Matter}

Quantum Observership aspires to be anti-metaphysical; but it is both epistemologically anti-realistic and metaphysically idealistic. Thoughts and macroscopic sensory experiences are everything. No actual determinate world in itself exists below the level of macroscopic observables, not even one of primary properties; and there are no objective answers or scientific truths because there are no independently existing objects. In Quantum Observership, no objectively existing world of space/time/mass/energy exists; no atoms, protons, electrons, or other particles or wavicles exist objectively in and of themselves. Since they don't exist at all in themselves, they do not have even partly definite size, shape, position, weight, resistance, velocity, and momentum (which is mass multiplied by velocity). Thus, our questions about independent realities have no correct answers. Correspondence theories of truth and perception are rejected. Concepts, propositions, and sensations have no objective referents; they merely cohere with other concepts, beliefs, and experiences over time; or they fail to do so. Being consists in being perceived, nothing more. Truth consists in coherence with other beliefs, not with correspondence to reality.

Critical Realists, by contrast, think that a real world exists beyond our perceptions, and that it closely resembles the spatially extended and mathematically measurable qualities of our sensations. Scientific and cosmological beliefs refer to this real world. True empirical beliefs describe correctly an objectively existing world of space/time/mass/energy; false beliefs misdescribe it. In modern chemistry, electrons and protons in themselves are often said to lack secondary qualities of color, taste, odor, and sound; but they still have primary properties of size, shape, weight, resistance, velocity, and momentum. Yet, in critically realistic quantum physics, the objective reality of these properties cannot be exactly what they were conceived to be in pre-quantum classical physics.

Before quantum theory, classical particle physics was Newtonian in spirit. The primary properties of subatomic particles were thought to be exactly like those of billiard balls, only smaller. All the way down to the smallest particles, Newtonian matter in itself possessed primary qualities in a fully definite and determinate manner. By contrast, in Quantum Critical Realism, some of the primary properties of quantum matter exist only indefinitely, indeterminately, by degrees, and relative to prehenders or measurers (who need not be conscious beings).

The Whiteheadian concept of "prehension" is fruitful and illuminating in understanding quantum matter. Prehending is the temporal process of grasping something or taking it in experientially. Prehenders are temporally experiencing or prehending subjects or momentary occasions of experience that take data from past events into themselves and actively process this received information in a variety of ways. Perishing past events causally transmit or imbue what they can of themselves into their successors, which actively receive, integrate, and 
thus prehend that data into themselves. They make their own fleeting creative contribution to ongoing reality and perish themselves, to be pretended in turn by their own temporal successors.

Consider ten important contrasts between classical and quantum matter. They differ significantly with respect to (1) definiteness/indefiniteness of spatial extension, (2) definiteness/indefiniteness of position and motion, (3) definiteness/indefiniteness of location, (4) continuity/discontinuity of existence, (5) causal determinateness/indeterminateness, (6) internal vacuousness/sensitivity, (7) local/non-local causation, (8) absolute/relative spatial properties, (9) absolute/relative temporal properties, and (10)organic holistic unrelatedness/interrelatedness.

(1) Definiteness/indefiniteness of Spatial Extension. Classical electrons, protons, and other particles had fully definite spatial extension, that is, size, shape, and position, at all times; but quantum particles have merely a fairly definite size, shape, and position-but only when they lack definite momentum and velocity, and only when some measurement or prehension (which need not be conscious) somehow takes account of just those properties. When we know a particle's pace of passage, we cannot know its place. When it has a definite place, it cannot have a definite pace. Data about pace and place cannot be prehended simultaneously. Realistically construed, they cannot exist simultaneously. How we or other non-conscious prehenders try to "measure" for them partly determines what they can be.

(2) Definiteness/indefiniteness of Position and Motion. Classical physical particles had a fully definite and discoverable velocity and momentum at all times, but quantum particles have merely a fairly definite velocity and momentum at best-but only when they lack full definiteness in size, shape, and position, and only when something prehends, takes account of, or experiences just those aspects of their motion. When we prehend a particle's place, we cannot know its pace; particles cannot be, or be prehended as, simultaneously definite in both ways. The idea is odd, but physical reality just is odd. Things are what they are only interrelationally; but these interrelations exist objectively, not merely within consciousness, says Quantum Critical Realism

(3) Definiteness/indefiniteness of Location. Classical physical particles had what Alfred North Whitehead called "simple location," a fully definite, determinate, and independent spatial position or locus at all times, but quantum physical particles behave like "wavicles," being spread or smeared out over small but indefinite regions of space at any given time and at different times. Particle aspects of quantum-level entities that constitute societies of successive events are always accompanied and guided by wave aspects. In two-slit experiments, the waves go through both slits, even though the particles go through only one. String theorists speculate that the smallest and most basic constituents of matter are very short and small looped (in most versions) strings of energy. Every string, qua string, in every kind of particle is exactly like every other; and 
differences between kinds of particles result from differences in the vibrational frequencies and amplitudes of the tiny strings in their cores. ${ }^{19}$ String theory, currently unverified, says that all the observed properties of the physical world can be explained if under different conditions these too-small-to-be-observed strings undulate, ball up, tie knots, disappear and reappear, resonate harmoniously (or disharmoniously) with other strings, and are otherwise sensitive to their environments. These issues need not be settled here; at present, string theory is little more than an intricate mathematical game with little or no empirical grounding. For the moment, we note simply that rapidly and constantly vibrating strings, or smeared out particles and wavicles, could never have the definite independent locus of substantively enduring pointlike classical particles. They may essentially lack other kinds of definiteness as well.

(4) Continuity/discontinuity of Existence. Classical physical particles were thought to exist without interruption and to move continuously through the infinitely divisible continua of Newtonian space and time. Electrons, for example, moved smoothly through the infinite number of points into which their supposedly perfect circular orbits are divisible. By contrast, quantum particles exist and move only discontinuously from place to place without passing through the intervening spaces, and the orbits to which they are restricted are broad shells, not perfect circles. When a photon is added to an orbiting electron, the "excited" electron prehends this addition, then jumps up to the next allowable orbit-like region without traversing the quantum-forbidden space that separates allowable orbits. Then, almost immediately, but we can't predict exactly when, it may throw off the photon and jump back down without passing continuously through the intervening space between orbit-like-shells. Something similar happens when an electron or any other particle moves forward within its own orbital shell or quantum-permitted region. Its particle features move forward as so many periodic and discontinuous pulsations of energy, unpredictably here, unpredictably there, somewhere within the tiny region of its orbit-like shell, but never in an exactly circular or linear path. Whitehead, who neglected its wave-like features, compared an electron's orbit to the travels of an automobile that appears only at milestones but nowhere in between $!^{20}$ Sten Oldenwald says that physical particles play "a hop-scotch game to avoid gaps where spacetime doesn't exist." ${ }^{\text {"1 }}$ Physical particles endure through time only as so many successively pulsating repetitions and ongoing prehensions of their particular kind of particle/wave/string patterns-electron forms, proton forms, photon forms, and the like.

(5) Causal Determinateness/indeterminateness. Causal determinism was believed to be complete for classical physical particles. In rigid conformity to exact and efficacious physical laws, all motions and changes made by classical physical particles followed exactly from necessary and sufficient antecedent formal and physical conditions and were in principle susceptible to absolutely precise mathematical measurement. If the positions and velocities of all particles 
were known, everything could be predicted. By contrast, quantum particles, lacking absolute positions and velocities, are not fully and predictably determined by and knowable from quantum laws and antecedent physical conditions. Every mathematically measurable quantity of physical entities is susceptible to unpredictable fluctuations or perturbations. Neither we nor an ace predictor like God can tell or predict when individual particles will change orbits, exactly where they will appear next within broad-band orbital shells, which slits they will go through, the directions in which they will fly when scattered, or exactly which ones will decay and produce atomic radiation. Quantum uncertainty, indeterminateness, and spontaneity pervade all physical reality. By their erratic behavior, vast numbers of individuated quantum-size entity/events create quantum laws, which merely summarize formally and probabilistically both their collective regularities and their individual eccentricities. Formal laws as such exert no efficient causality of their own. The laws of nature are epistemologically useful but ontologically powerless.

(6) Internal Vacuousness/sensitivity. Classical physical particles were internally inert, solid, vacuous, and fully actual; but quantum wave/particles are at best only partly determinate internally, and they are environmentally sensitive and reactive. In part, they are indeterminate sets of possibilities in process of becoming fully actual. Each of a wavicle's fleeting pulsations of energy in some unconscious way takes on or prehends a determinate form (electron, photon, and so on) inherited from immediately past pulsations, and each projects this form into the future as it perishes in time. Wave/particles are not internally inert, solid, impenetrable, and vacuous; they take their own immediate constitutions and past and future environments into account in deciding what to do or what to become next. All physical wave/particles experience and are internally sensitive to their external environments, at least unconsciously. They know what kind of wave/particles they have been in the past. As they confront the future, they internally take into account not only their own past but that of their wider environment. What wavicles are and what they do is largely constituted by their relations. Protons, neutrons, electrons, and other particles know when they are and when they are not located within atoms; they know one another's natures and whereabouts; and they behave themselves accordingly. Photons, electrons, and other wave/ particles seem to know whether or not they are surrounded by and partly composed of waves; they respond actively when photon quanta are attached to themselves; they are aware of how their twin particles are spinning. When they are confronted by the environmental prospect of going through either one slit or two, they know the difference in advance and behave accordingly.

Based on earlier work by Louis de Broglie and David Bohm, John S. Bell offers a highly plausible realistic but relational solution to the particle-wave dualism inherent in the two slit experiment: 
A particle passing through just one of two holes in a screen could be influenced by waves propagating through both holes. And so influenced that the particle does not go where the waves cancel out, but is attracted to where they cooperate. This idea seems to me so natural and simple, to resolve the wave-particle dilemma in such a clear and ordinary way, that it is a great mystery to me that it was so generally ignored. ${ }^{22}$

The idea that all elemental particles are accompanied and perhaps even guided by waves is now widely accepted in quantum physics, as expressed in the very concept of "wavicle." Realistic objectivists like David Bohm and B. J. Hiley completely repudiate the wave-function-collapse account of quantum-level definiteness. They contend, probably correctly, that most of the dither about definiteness resulting only from collapsing wave functions just ignores the fact that wavicles are particles as well as waves. Wave collapse conundrums assume incorrectly that in themselves wavicles are nothing more than indeterminate waves and forget that they are also particles bearing their own definiteness. ${ }^{23}$ Bohm and Hiley contend that in a two slit experiment, the particle definitely goes through one slit, we know not which, while the accompanying wave goes through both slits..$^{24}$

(7) Local/non-local Causation. Classical Newtonian material particles were also externally inert, naturally at rest, utterly incapable of self-initiated motion or of being moved by distant entities. They could be moved only mechanically by other external physical entities with which they were in direct physical contact. Both Newton and Einstein were horrified by the thought of what Einstein called "spooky action at a distance."

But isn't gravity action at a distance? Not for Newton! Gravity, for Newton, was not a physical force; it was a divine force. It was a manifestation of God's direct activity in moving non-contiguous objects lawfully in relation to one another. Through gravity, God moves otherwise immovable material objects that are separated from one another by distance. ${ }^{25}$ Later atheistic Newtonians conveniently ignored this feature of classical Newtonian matter/gravity. Einsteinian gravity also involves no action at a distance; it consists of curvature of space emanating directly and with continuity from mutually attracting entities.

In contemporary quantum physics, wavicles are neither externally inert nor internally vacuous. They never exist in inert independence. Action at a distance is allowed, indeed required. Wavicles are definitely not internally vacuous. Information is integral to their internal existence, for they can somehow anticipate the presence of two slits before they get there. They are aware of open alternatives and in some primitive way can choose among them. In quantum non-locality (explained more later), information about what happens to one wavicle is instantaneously transmitted to and registers with another, perhaps at great distances. 
(8) Absolute/relative Spatial Properties. Classical particles existed in absolute Newtonian space and time, which were supposed to be uniformly the same throughout the whole universe. By contrast, when combined with relativity theory, quantum particles have only a relativistic locus in Einsteinian spacetime. They are constituted by their spatiotemporality. Their primary spatial properties of size, shape, resistance to change, position, and momentum vary immensely, depending on how other events prehend them and on the speed with which they and their accompanying spacetime frames are being accelerated through the universe. A spaceship accelerated to the speed of light would flatten out; its mass would increase to infinity; and it would offer infinite resistance to further increases in speed, which is why acceleration to or beyond the speed of light is so implausible. Approximations to the speed of light would be approximations to infinity. No matter what its speed, a Newtonian spaceship, by contrast, would always have exactly the same size, shape, mass, and inertia; but overwhelming evidence now indicates that ours is not a Newtonian world.

Aristotle defined change or motion as "transition from potentiality to actuality," but this idea never gave rise to a single mathematical formula that empowers human beings, scientists in particular, for prediction and control. Modern mathematical physics began when Galileo replaced Aristotle's qualitative definition with a quantitative definition. Motion or velocity equals the space or distance traveled divided by the time required for the trek: $v=s / t$. Thus, if we travel a hundred miles in two hours, our speed or velocity is fifty miles per hour; and if we travel sixty miles in three hours, our velocity is twenty miles per hour. This formula, in which velocity varies with differences in time or distance, works perfectly well with everything except the speed of light. The speed of light in an invariant absolute in an otherwise relativistic universe.

Toward the end of the nineteenth century, physicists James Clerk Maxwell and Albert A. Michelson considered the speed of light and made some remarkable discoveries that paved the way for Einstein's insight that light is a universal constant and does not vary in speed with variations in distance and time. Because the speed of light is constant, space and time cannot be Newtonian absolutes, so Einstein proclaimed in his "Special Theory of Relativity" in 1905.

Time and temporal processes would proceed at the same pace everywhere and at every speed if Newton had been right. In fact, however, one earthborn twin traveling for an interval on a spaceship at close-to-light speed would be much younger when she returned than the twin who remained on earth. The physical clocks in her spaceship and her own biological clocks would slow down in proportion to accelerated speed. The speed of light can be constant at all speeds only if primary properties of size, shape, mass, resistance, mass, and time itself vary with speed. Classical matter possessed primary properties absolutely; but collectively if not individually, quantum matter possesses them only relative to acceleration. Time slows down and mass increases as velocity increases, and its pace is faster as mass and velocity decrease. 
Since the pace of time itself is affected by the speed with which a spacetime frame flows through the universe, are our cosmological calculations concerning the age of the universe affected? Would other observers at other speeds get more than, or less than, fifteen billion years for the age of the universe? Does the universe have this age only from our own relative vantage point? Probably so, in answer to all of these questions; but the world of nature manifests much more uniformity than popularizes of relativity often lead us to believe. We have learned in recent years that a uniform microwave background is universally accessible; and its constancy could function as a uniform frame of temporal reference for all space travelers. ${ }^{26}$ Light is not the only form of energy with the constancy of the speed of light; all electromagnetic radiation travels at that speed. Quantum non-locality, which we haven't learned to put to practical use, instantly connects distant parts of the universe. The basic structures and laws of nature are the same no matter how fast anyone is going.

Any observers moving at any speed anywhere in the universe should be able to view the overall structure of the universe and determine that it came into being at some point in the finite past. Without being able to correlate their watches and their calculations perfectly, all intelligent beings within the universe should be able to discover the Big Bang and conclude that our universe was created a finite while ago.

The universe-wide instantaneous action at a distance of quantum nonlocality restores additional physical meaning to cosmic simultaneity, despite our inability to use non-locality to send instant messages to observers elsewhere in the universe. Still, all intelligent observers scattered throughout the universe should be able to discover that the Big Bang happened, though if they could communicate they might not be able to agree about precisely when it happened.

(9) Absolute/relative Temporal Properties. Classical matter possessed its spatial properties in complete independence of time. Newtonian space and time were completely independent of one another and of the material masses within them. If the temporal duration of an electron were cut infinitesimally thin, it would still fully possess its definiteness of spatial size, shape, motion, mass, and so forth. But the complete independence of time, space, and mass do not apply to quantum/relativity matter. Minimal finite temporal durations, what Whitehead called "specious presents," are required for the existence of any spatial properties at all, and minimal spatiality is required for the existence of any temporality at all. Spaces and times can be sliced infinitely thin by imagination or calculation, but no infinitesimals can actually exist. Nothing can really exist for less time than Planck time $\left(10^{-43}\right.$ second $)$ or in less than Planck space $\left(10^{-33}\right.$ centimeters). To exist spatially as matter, as extended stuff, entities must also have some temporal or durational properties. In contemporary physics, time and space are inescapably interdependent, not totally independent as they were in classical physics. 
10. Organic Holistic Unrelatedness/interrelatedness. Classical physical particles are what they are quite independently of all else. Since they exist contingently, they require causes; but they do not require anything else to be what they are. They can be isolated and studied quite independently of their relations with other things. By contrast, in quantum world-views, both microscopic and macroscopic realities are what they are by virtue of their internal relatedness with other things with which they form interdependent wholes. Nothing requires only itself to be what it is. All properties are relational properties; holistic relations to other things are internal to the constitutions of all realities. All previously identified traits of matter involve organic relational wholes in which they parts are what they are by virtue of the measuring or pretending wholes within which they exist. Smeared out quantum-level entities have only relative but not absolute definiteness of spatial extension. When measured or pretended for definiteness of position and motion they have them separately but not together. Their precise location is relationally definite by degrees. They exist discontinuously in relational spurts. They are partly caused by the externalities that they internalize and are partly self-caused. They are internally sensitive and responsive to their surroundings. They are partly what they are by virtue of local and partly by non-local causation. They are constituted by their spatial and temporal relations, and these condition all their other properties.

Thus, even matter is not what it used to be anymore! Commenting on Gilbert Ryle's characterization of mind/matter dualism as "the ghost in the machine," Paul Davies and John Gribbin tell us that "Today, on the brink of the twenty-first century, we can see that Ryle was right to dismiss the notion of the ghost in the machine-not because there is no ghost, but because there is no machine."'27 Today we see clearly that matter in the classical sense just does not exist at all. A critically realistic theory of perceptual correspondence to objective reality must be adjusted to take account of quantum/relativity physics. It must emphasize both the definiteness within holistic interrelatedness of, and the partial but not total spontaneity, indefiniteness, indeterminateness, and unpredictability of, the depths of nature that physics has unearthed.

Once proper adjustments have been made, matter still exists objectively and retains a great deal of definiteness in and of itself alongside its unpredictable self-creativity. Despite relativity physics and quantum measurement problems, as Victor J. Stenger notes,

Many properties of matter are fixed and, for practical purposes permanent. They can be determined without their respective measurements interfering with one another. These include rest mass, electric charge, magnetic momentum, and spin. Material bodies possess many unambiguous features that are not the slightest bit ephemeral. ${ }^{28}$ 
In a similar vein, Holmes Rolston, III indicates,

We must not be overwhelmed with this relativity of everything, for many things are not observer-dependent. The equations, the basic laws are invariant. Some of the physical constants of nature-the speed of light, the charge on an electron, or the number of atomic shells, the atomic table, chemical reactions, and so on-will presumably be the same for all observers, as will ordinarily be the order of succession of causally related events. Thus, there is considerable objectivity in relativity theory. ${ }^{29}$

A partly or critically realistic interpretation of the physical world is possible if the primary properties of unobserved sub-atomic entities and processes are to some degree interrelatedly determinate apart from consciousness and transmit that determinateness to their successors, including our conscious sensory experiences. In response to those quantum experiments indicating that observation of one photon affects the spin of another photon, Roger Penrose, who declares himself to be a realist,,$^{30}$ says,

The best suggestion that I can make at this stage would be for a picture involving some sort of partially formed, partly bifurcating spacetime, where the nature of the spacetime has not been adequately resolved until the second photon observation has taken place. ${ }^{31}$

If the contents of spacetime are partly formed apart from conscious observation, and if they correlate to that extent with conscious perception or prehension, partial or Critical Realism is correct. Quantum Critical Realism, adapted to quantum/relativity physics, still allows enough definiteness in objectively existing nature to account for cosmic and biological evolution. This is probably the best available argument for the truth of Critical Realism.

Most physicists have not abandoned realism for an idealistic metaphysics, despite the advances and challenges of quantum mechanics. Most cosmological theories are to some degree realistic. The Standard Model of Big Bang Cosmology assumes that an objectively existing universe of space/time/mass/energy came into being between ten and twenty billion years ago. The Inflation modification makes the same assumption but tries (successfully?) to make it more plausible. Infinite universe theories like Steady State and Plasma Cosmology affirm that an objectively existing universe of space/time/mass/energy is literally infinite-in itself-not just in our perceptions, thoughts, and theories. Antecedent Universe Cosmologies postulate objectively existing universes that preceded and caused our own. World-ensemble theories postulate an objectively existing Superspacetime that gives birth to many objectively co-existing universes, some perhaps erupting or branching from others. Most quantum and relativity physicists and scientific cosmologists agree that space/time/mass/energy are insepara- 
ble; but they do not believe that they exist only in our experiences, thoughts, and theories. Quantum Observership contends that this quasi-realistic approach is all wrong; but for many reasons like those given in the preceding discussion, most physicists think that Quantum Observership is all wrong.

A few dissenting scientists and philosophers of science have subjectivist leanings, at least in their weaker moments. In 1983, a younger Paul Davies expressed antirealism when he wrote that "The commonsense view of the world, in terms of objects that really exist 'out there' independently of our observations, totally collapses in the face of the quantum factor." ${ }^{32}$ In some of his moods, the early Albert Einstein surmised that space and time are ultimately unreal and exist only as human illusions. He came to this conclusion, not on scientific grounds, but because he subscribed to a Spinozistic metaphysics. Kantian idealism would give the same results. Later, Einstein vehemently opposed the idealistic, anti-realistic implications of Quantum Observership. ${ }^{33}$

Quantum Observership gives quantum mechanics a robustly anti-realistic flavor, but most masters of quantum mechanics are realists. To the suggestion that we should just follow the rules of quantum physics pragmatically without asking questions about reality or trying to form a picture of reality, Roger Penrose responds: "This seems to me to be wholly unreasonable. Physics, after all, constitutes our best way of groping for the true nature of the real world in which we find ourselves." ${ }^{\text {"34 }}$ Bernard d'Espagnat defines Realism as "the doctrine that regularities in observed phenomena are caused by some physical reality whose existence is independent of human observers. ${ }^{\prime 35}$ After thoroughly examining quantum experiments that intimate anti-realism, d'Espagnat concludes that abandoning realism

trivializes the entire scientific enterprise. Science is reduced to a set of recipes for predicting future observations from a knowledge of past ones. Any notion of science as "the study of nature" is a phantom. One can imagine a physics grounded on positivistic principles that would predict all possible correlations of events and still leave the world totally incomprehensible. Given the extreme consequences of abolishing realism, one is inclined to cling to this premise. ${ }^{36}$

Reconciling Critical Realism with quantum mechanics generates an odd picture of physical reality, as we saw in contrasting Newtonian with Quantum concepts of matter; but Realists are willing to pay the price. Physical reality just is odd, says quantum/relativity theory.

In 1964, John S. Bell demonstrated a fundamental incompatibility between the natural order of things disclosed by quantum mechanics and Einstein's insistence on "locality,"-the belief that there can be no faster-than-light causality. Einstein called faster-than-light causality "spooky action at a distance." Bell insists, contra Einstein, that "Events at one place propagate to other places faster 
than light. This happens in a way that we cannot use for signaling. Nevertheless it is a gross violation of relativistic causality. ${ }^{37}$ Many quantum theorists believe that non-local causality is the key to reconciling quantum theory with scientific Realism. Bernard d'Espagnat insists that messages really can travel faster than the speed of light. ${ }^{38}$ Roger Penrose affirms both that there are faster-than-light correlations between entangled events and that photons really can be (partly) in two places at once. ${ }^{39}$ David Bohm and Basil Hiley ${ }^{40}$ defend realistic and deterministic interpretations of quantum mechanics that allow for non-local fasterthan-light connections between events. Just before sending the final version of this book to my publisher, I discovered that John A. Jungerman explains nonlocality clearly and affirms it unequivocally on the basis of now abundant experimental evidence. ${ }^{41}$

Not all realists accept non-locality. In his very thorough but somewhat technical defense of quantum realism, Henry Krips rejects non-locality in favor of hidden variables..$^{42}$ In his very readable discussions of the topic, a skeptical Victor J. Stenger contends, "Now, after a series of precise experiments, the issue has been decided: hidden variables that are both local and real are ruled out" and "nonlocality exists only in theory" and cannot be confirmed experimentally. ${ }^{43}$ The question of non-local causation does not have to be decided here, but the case for it is much stronger than Stenger suggests. There is probably something to it, and it must be considered carefully by anyone who wishes to understand the interconnectedness of things.

Realistic quantum theory emphasizes the objective reality of fields as well as of waves and particles. Fields are objectively existing, invisible, colorless, regional sets of physical habits or dispositions with ill-defined borders; they require no medium of actualized waves or particles for their causal efficacy and reality; but they bear both information and energy; and these influence the wavicles within them. They inform, give form to, their components. Specialized regions, perhaps all regions, of space itself-some more than others-bear physical and formal properties that structure particle/wave events within themselves.

Finally, a rigid causal determinism appears to reign supreme at the level of macroscopic entities encountered in everyday experience to which Newtonian physics applies; but at the level of quantum systems, both indeterminateness and indeterminism are the rules. Quantum events in themselves and in our perception of them are neither fully determinate nor fully indeterminate with respect to their primary spatial and temporal properties. They are neither fully determined by nor left completely undetermined by their causal antecedents. Quantum Critical Realists are convinced that spontaneity is objectively real, not just an expression of human ignorance. Einstein was wrong when he decreed that "God does not play dice with the universe!" The universe plays dice both with itself, and with God. 


\section{ii. Observership and Causation}

A plausible scientific account of human origins must affirm that relatively definite physical processes existed objectively for billions of years before conscious observers evolved; yet, according to Quantum Observership, conscious observers contribute all definiteness to what is observed, and nothing unobserved exists at all. Quantum Observership must avoid this logical predicament, which it cannot do; it must also explain the magical causal process by which conscious observation affects or creates physical entities and processes. Is all seemingly physical causation just another example of spooky action at a distance without a physical medium? Is energy really exchanged between observer and observed? Do conscious observers as such project photons or radiant energy onto other photons and particles to collapse their infinite potencies into definite actualities of position or momentum? If so, could the projected photons or radiation be detected? Would this energy have enough definiteness of its own when not being observed to do its work? Or, as idealist Jonathan Edwards held, does God cause everything directly, while events and beings within the world, including human observers, cause nothing? Or do human observers without God simply create photons, electrons, other particles, and the universe itself ex nihilo in the very acts of looking for and finding them? How does this happen? Do conscious observers create the Big Bang in the very act of perceiving or conceiving the evidence for it, in something like the way in which God, in some versions of fundamentalistic "Creation Science" directly creates the fossilized bones of dinosaurs that never existed? If so, is Quantum Observership any more plausible than magical Creationism?

\section{iii. Ambiguities Involving "Observer" and "Measurement"}

In Quantum Cosmology, the meanings of "observer" and "measurement" are unclear. Quantum physics appears to divide the universe decisively into (1) observers (2) measuring instruments, and (3) observed or measured quantum events; but these are not sharp distinctions. What is an observer? Must all observers be conscious entities? Are observers themselves composed of quantum events? Are their instruments composed of quantum events?

In Quantum Observership, observers must be conscious-like human beings. Perhaps a conscious animal, a dog, or even Schrödinger's cat would do, but this is not always clear. What if a person instead of a cat had been in Schrödinger's box? Why couldn't the observer be an omni-observant God who, presumably, would always confer as much definiteness upon the world as it needs? Most physicists prefer not to appeal to God's existence or to consciousness to solve physical problems. God might provide a perfect solution to the puzzles of Quantum Observership if most quantum physicists were not biased against Theism. Yet, conscious divine, human, or animal observers may not be 
necessary at all for conferring definiteness upon quantum events. We cannot simply equate "observer" with "consciousness."

In realistic interpretations of quantum theory, observers need not be conscious beings. Anything responsive to its environment will do. According to Werner Heisenberg, "It does not matter whether the observer is an apparatus

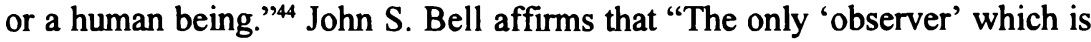
essential in orthodox practical quantum theory is the inanimate apparatus which amplifies microscopic events to macroscopic consequences. ${ }^{245}$ Observers may be unconscious measuring machines like Geiger counters or photographic plates.

Definiteness-conferring observations need be nothing more than unconscious physical processes taking account of or interacting causally and prehensively with others. Scientific instruments functioning as unconscious observers are themselves composed of quantum-level physical processes. No sharp line separates microscopic and macroscopic processes and observers, even though our senses are generally responsive only to quantum-level events en masse. Victor J. Stenger indicates that detectors "need not be limited to the sensory apparatus of human beings or their scientific instruments. The term 'detector' can also encompass the particles in the environment surrounding the system." ${ }^{346}$ Thus, one physical process pretending or interacting responsively with another is all that is required to confer physical definiteness. If all physical processes interact sensitively with other physical processes, the universe in itself, quite apart from conscious observers, would have all of the exactitude that Critical Quantum Realism attributes to it. As Abner Shimony puts it:

When a physical variable which initially is merely potential acquires a definite value, it can be said to be actualized. So far, the only processes we have mentioned in which potentialities are actualized are measurements, but in a non-anthropocentric view of physical theory the measurement process is only a special case of the interaction of systems, of special interest to scientists because knowledge is thereby obtained, but not fundamental from the standpoint of physical theory itself. ${ }^{47}$

Heisenberg, Bell, Stenger, Shimony, and other Quantum Critical Realists think that there is nothing special about either conscious observers or measurements. Popularizers of quantum mechanics like Paul Davies ${ }^{48}$ contend that definiteness is achieved at the level of microscopic wave-function events only as they collapse when measured by conscious observers using macroscopic measuring and recording instruments. Schrödinger's cat really is in a dual alive-dead wavefunction state until a human observer reads the Geiger counter. John Bell, by contrast, maintains that notions like "observer" and "measurement" are so obscure that they cannot be fundamental for physics, and that there is no sharp line of demarcation between the microscopic and the macroscopic. ${ }^{49}$ Elemental 
reflection will show that Bell is right. John Wheeler himself eventually came around:

Let us not invoke either "consciousness" or "observer" as prerequisite for what in quantum mechanics we call the elementary act of observation. What counts as "observation" for the purposes of quantum mechanics is the irreversible act of amplification. It may not be clear how much amplification is required. ${ }^{50}$

The quantum-level domain seems to confer degrees of definiteness upon itself merely through causal interactions between quantum events, occasionally at speeds as fast as light, but usually not. Physical causation is always temporalistic and prehensive, but it is not rigidly deterministic.

A significant degree of both definiteness and indefiniteness could exist in quantum events if, as Process Philosophy affirms, partly indeterminate events in their present moment of immediacy prehend, take account of, and in that sense measure, immediately past events that become fully definite only as they perish and are prehended by their successors. Demarcations between eventdurations need not be as sharp as process thinkers previously assumed, as explained later. The present occasion in any causal cone of spatiotemporal events possesses a high degree of internal freedom, self-creativity, and indeterminateness, mixed with degrees of definiteness or data inherited from the past. Events acquire their fullest definiteness as they complete themselves, perish into the past, and are perceived or prehended (usually unconsciously) by their partlyself-creating successors. In physical terms, wavicles are always highly definite in themselves; but they acquire a more complete but still very similar definiteness as they perish in time and are succeeded and "observed" by their immediate temporal successors.

Quantum theorists recognize that a measurer must be something spatially distinct from the object measured, but temporal distinctness must also be emphasized. All causation from space to space is temporally ordered. Also, according to Process Philosophy, all mentality is spatially ordered and extended; and no totally disembodied mentality exists anywhere. All events at every level of existence have both mental and physical poles, but physical poles are both spatially extended as well as causally responsive and efficacious. Even God is embodied in the world, or some world, and is not a purely incorporeal disembodied spirit.

If all immediately successive events are observers or prehenders of their predecessors and receive forms and data from them, the unconscious world of nature in itself has sufficient definiteness to exist in itself and to evolve complex conscious observers like us; and each relatively independent and partly selfcreative present moment at every level of complexity has sufficient indefiniteness to allow for ubiquitous freedom and creativity. 
In Process Philosophy, the partial indeterminateness of present events extends to all levels of reality, no matter what their degree of organization and complexity. Atomic and sub-atomic uncertainty is not the same thing as free will and creativity in immensely complex human subjects, but both are manifestations of a universal principle of creativity; and human creativity supervenes upon physical indefiniteness. Newtonian mechanistic determinism excluded human freedom, but quantum level uncertainty makes room for it without being identical with it. Natural processes become more and more fully determinate as decisions are made and information is synthesized in the present, as events perish into the past, and as their successors perceive or prehend them. Temporalistic entities receive as much as they can from their predecessors and transmit what they can of themselves into their successors. These transmitted forms and data give continuity, memory, and relative self-identity through time to chains or temporaliy ordered societies of consecutive spatiotemporal events, ranging from quark-pulsations to streams of human consciousness.

Our own conscious present moments of receptivity, partial self-creativity, relative independence, subjective immediacy, and self-enjoyment are partly indefinite, but not infinitely indefinite. Degrees of definiteness and indefiniteness are equally real at all levels of natural complexity. Photon wavicles definitely cannot collapse into electrons; electron pulsations cannot collapse into proton or neutron pulsations; and streams of human consciousness cannot degenerate directly into streams of canine or bovine consciousness. Each partly determinate particle/wave includes a very limited set of unactualized possibilities. Where will it jump to next? Where exactly will it go when scattered? Through which slit will it pass? When will it "decay" into a free particle?

Events become determinate partly by inheritance, partly by decisions of the moment among open possibilities, and partly by perishing in time to be prehended by their successors. Quantum indefiniteness versus measurement is nothing more mysterious than causation between immediately past and present spatiotemporal occasions. That in itself is mysterious enough!

To summarize, Quantum Observership argues from unusual interpretations of perplexing experiments in quantum physics to an Idealistic Metaphysics that denies the objective existence of space/time/mass/energy. No physical objects exist without conscious physicists or observers of some sort, says Idealism. In itself, the physical world is merely an infinite set of indeterminate possibilities for perception; and the determinateness of the physical world consists in and is caused or created by its being perceived or observed.

Quantum Observership is incompatible with cosmic and biological evolution. It generates the unresolved paradox that observers create the Big Bang and the evolutionary process, but the evolutionary process and the Big Bang create all observers. It cannot explain the magical causal procedure by which observers confer determinateness on physical entities and processes, including those in the distant past. As in Kantian Idealism, Quantum Observership reduces space, 
time, energy, and causation to forms of experience that have nothing to do with things in themselves or objective realities. Most modern scientists reject Quantum Observership and its insistence that space/time/mass/energy exist only in human consciousness; they strongly prefer, with good reason, the partly realistic theory that the physical world exists objectively, independently, but relationally. Yet, the relations need not be with conscious observers.

Finally, the notions of "observer," "instrument," and "measurement" cloak serious ambiguities. The referents of all of these concepts are partly composed of quantum-level events. If observers are allowed to be unconscious physical objects, including measuring instruments, and if no sharp divisions exist between the macroscopic and the microscopic or between minds and matter, and if measuring is merely causal interaction, the mutual interconnectedness of effects and causes, then in the absence of conscious observers, the physical world can prehend, observe, measure, and confer sufficient determinateness upon itself to account for the objective reality of the Big Bang and for the evolution of nature prior to the emergence of consciousness.

A Kantian/Copernican idealistic revolution in epistemology is just as anthropocentric as the geocentric theory that placed humankind at the geographical center of the universe. A realistic nonanthropomorphic natural science affirms that we have our being and our becoming within and as a part of a vast, natural, objectively existing, vibrant universe of interdependent, partly determinate, partly indeterminate pulsations of space/time/mass/energy. For billions of years, this independently existing system of nature possessed a high degree of definiteness in itself; unconscious prehensive relational responsiveness is ubiquitous. In our part of the Milky Way, no conscious observers existed before animals, including our own most primitive evolutionary ancestors, emerged from the primordial terrestrial slime. We exist within and as a part of the totality of objective spacetime. Nature does not exist merely within us as mind-dependent conscious perceptions to which nothing objective and determinate corresponds. The objective existence of highly (but not totally) determinate space/ time/mass/energy makes a very practical difference. Without it, we would not be here at all. With it, Quantum Observership is wrong. 